\title{
Selective tuning of spin-orbital Kondo contributions in parallel-coupled quantum dots
}

\author{
Heidi Potts $\odot,{ }^{1, *}$ Martin Leijnse, ${ }^{1}$ Adam Burke $\odot,{ }^{1}$ Malin Nilsson, ${ }^{1}$ Sebastian Lehmann, ${ }^{1}$ \\ Kimberly A. Dick, ${ }^{1,2}$ and Claes Thelander ${ }^{1, \dagger}$ \\ ${ }^{1}$ Division of Solid State Physics and NanoLund, Lund University, SE-221 00 Lund, Sweden \\ ${ }^{2}$ Centre for Analysis and Synthesis, Lund University, SE-221 00 Lund, Sweden
}

(Received 11 December 2019; accepted 9 March 2020; published 30 March 2020)

\begin{abstract}
We use cotunneling spectroscopy to investigate spin, orbital, and spin-orbital Kondo transport in a strongly confined system of InAs double quantum dots that are parallel coupled to source and drain. In the one-electron transport regime, the higher-symmetry spin-orbital Kondo effect manifests at orbital degeneracy and no external magnetic field. We then proceed to show that the individual Kondo contributions can be isolated and studied separately: either by orbital detuning in the case of spin Kondo transport or by spin splitting in the case of orbital Kondo transport. By varying the interdot tunnel coupling, we show that lifting of the spin degeneracy is key to confirming the presence of an orbital degeneracy and to detecting a small orbital hybridization gap. Finally, in the two-electron regime, we show that the presence of a spin-triplet ground state results in spin Kondo transport at zero magnetic field.
\end{abstract}

DOI: 10.1103/PhysRevB.101.115429

\section{INTRODUCTION}

The Kondo effect is a widely studied many-body phenomenon that has increased the understanding of strongly correlated electron systems. Experimentally, it can be investigated using quantum dots (QDs) with highly transparent tunnel barriers and manifests as a zero-bias conductance resonance. Most studies focus on the spin-1/2 Kondo effect, where an unpaired spin is screened in the absence of a magnetic field [1-3]. Two-electron spin states represent another common system for Kondo studies, where resonances arise from a vanishing singlet-triplet exchange energy [4-6], a magnetic-field-induced singlet-triplet crossing [7-12], or a triplet ground state [13-15]. More recently, the orbital Kondo effect, which relies on two degenerate orbitals, has received considerable attention. In particular, by combining spin and orbital degrees of freedom, the SU(4) Kondo effect can be studied [16]. The presence of such a higher symmetry can be identified by different temperature scaling [17] and an expected enhancement in the shot-noise properties [18-20].

In single QDs, orbital and spin-orbital Kondo transport has been observed in carbon nanotubes [18-23] and siliconbased devices [24,25]. However, in such systems, the orbital degeneracy is an inherent material property and the tunability of the orbital alignment is therefore limited. An alternative approach is to fabricate two parallel-coupled QDs for which

\footnotetext{
*heidi.potts@ftf.lth.se

†claes.thelander@ftf.lth.se
}

Published by the American Physical Society under the terms of the Creative Commons Attribution 4.0 International license. Further distribution of this work must maintain attribution to the author(s) and the published article's title, journal citation, and DOI. Funded by Bibsam. the orbitals can be tuned independently. Typically, this is realized in two-dimensional electron gases [17,26-30], but at the expense of limited Zeeman splitting of spin states.

In this work, we use parallel-coupled quantum dots in indium arsenide (InAs) nanowires to investigate the spin and orbital Kondo effect. We show that each degeneracy here can be induced and lifted selectively, which makes it an ideal system for studies of higher Kondo symmetries. We start by discussing Kondo resonances in the one-electron regime and present results on the spin, orbital, and combined spin-orbital Kondo effect. The individual contributions to the transport are isolated by electric-field-induced orbital detuning and magnetic-field-induced spin splitting. Furthermore, by controlling the interdot tunnel coupling $t$, we demonstrate that the formation of hybridized states inhibits the orbital Kondo effect. If $t$ is small, the energy gap of the avoided crossing can only be resolved if the spin degeneracy is lifted. This underlines the importance of isolating different Kondo mechanisms when studying their contribution to Kondo effects of higher symmetry. Finally, we investigate the Kondo effect in the two-electron regime when each of the QDs contains one unpaired electron, and a finite tunnel coupling between the QDs gives rise to two-electron states. A Kondo resonance at zero magnetic field is observed here due to a spin-triplet ground state (GS).

\section{EXPERIMENT}

Our study is based on InAs nanowires where a QD is defined by a thin zinc-blende (ZB) section between two wurtzite (WZ) barriers [31]. Figure 1(a) shows a schematic representation of the nanowire and a scanning electron microscopy (SEM) image of a representative device. Metal contacts (Ni/Au $25 \mathrm{~nm} / 75 \mathrm{~nm}$ ) are placed on the outer ZB sections as source and drain [32]. Transport measurements are performed in a dilution refrigerator with an electron temperature 

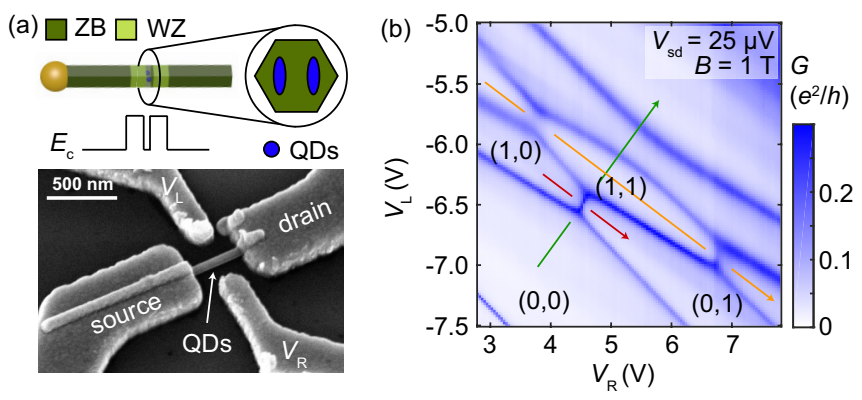

FIG. 1. (a) Top: Schematic of the nanowire and conduction-band alignment. Bottom: $45^{\circ}$ tilted SEM image of a representative device. (b) Conductance $(G)$ as a function of sidegate voltages at $B=1 \mathrm{~T}$. The electron populations on the left and right QD $\left(N_{\text {left }}, N_{\text {right }}\right)$ after subtracting $2 N$ electrons are indicated. Important gate vectors are shown in red, green, and orange.

$T_{e} \approx 70 \mathrm{mK}$. If applied, the magnetic field $B$ is aligned perpendicular to the substrate plane in this study. Previous studies have shown that two sidegates $\left(V_{\mathrm{L}}, V_{\mathrm{R}}\right)$ and a global backgate $\left(V_{\mathrm{BG}}\right)$ allow one to split the QD into two parallelcoupled QDs, and control the electron population on the two QDs independently. The system is highly tunable and the small effective electron mass provides large intradot orbital separations [32-34]. In recent works, we have furthermore demonstrated the formation of ringlike states when coupling the QDs in two points, resulting in a vanishing one-electron hybridization energy [35], and a spin-triplet ground state when each QD contains an unpaired electron.

\section{RESULTS AND DISCUSSION}

In this paper, we investigate transport in a regime where two orbitals (one from each QD) cross in energy and interact, as shown in Fig. 1(b). The electron population of the left and right dot $\left(N_{\text {left }}, N_{\text {right }}\right)$ is indicated. Here, $2 N$ electrons were subtracted on both QDs, as the contribution of filled orbitals can be neglected due to strong confinement (a large-range overview measurement can be found in Appendix A). In such an orbital crossing, Kondo transport due to both spin and orbital degeneracies is possible. The spin- $1 / 2$ Kondo effect is expected when one of the QDs contains an unpaired electron, which is the case for transport involving the $(1,0)$ and $(0,1)$ orbitals. Assuming a vanishing hybridization energy for these two orbitals, we additionally expect an orbital Kondo effect where they cross in energy. Additionally, in the $(1,1)$ regime, two-electron spin states provide degeneracies that can also result in Kondo transport. In order to study these different types of Kondo origins, we will focus on transport along the gate vectors indicated in green, red, and orange in Fig. 1(b).

\section{A. Spin-orbital Kondo effect}

Figure 2(a) shows a measurement of differential conductance $d I / d V_{\text {sd }}$ versus the source-drain voltage $V_{\text {sd }}$, along the green gate vector indicated in Fig. 1(b). The gate vector is chosen such that the $(1,0)$ and $(0,1)$ orbitals are approximately degenerate along this vector in the one-electron regime. A zero-bias peak in the differential conductance can be observed within the outlined Coulomb diamonds corresponding to the one-electron $(1 e)$, two-electron $(2 e)$, and three-electron $(3 e)$ regimes. Next, we investigate the $1 e$ regime in more detail by detuning the orbitals of the left and right QD with respect to each other (red gate vector). Due to the large interdot Coulomb energy $U_{1,2} \approx 3 \mathrm{meV}$, the system holds one electron along the whole vector, and all transport features are related to cotunneling events. The transport measurement at $B=0 \mathrm{~T}$ in Fig. 2(b) shows a zero-bias peak [36], as well as a step in differential conductance that approaches zero bias at zero detuning. Comparing with a schematic representation of the states and resulting onset of cotunneling transport [Fig. 2(c)], we can distinguish two transport processes: (1) the spin-1/2 Kondo effect on the populated QD and (2) cotunneling involving both QDs, which we will refer to as orbital cotunneling. For the latter, an electron tunnels out of the left (right) QD and is replaced by an electron on the right (left) QD (with or without a spin flip), and the energy cost of this process corresponds to the detuning of the two QDs. At zero detuning of the QDs $\left(\Delta E_{\text {orb }}=0\right)$, the onset of orbital cotunneling crosses zero bias. However, without further investigation, it is unclear whether both the spin and the orbital degeneracy contribute to the Kondo resonance.

Transport measurements at different temperatures were performed in order to extract the Kondo temperatures in different locations in the honeycomb diagram. The intensity of a Kondo zero-bias peak shows a characteristic decay with temperature, which can be described by the phenomenological expression $[1,37]$

$$
G(T)=G_{0}\left[1+\left(2^{1 / s}-1\right)\left(\frac{T}{T_{\mathrm{K}}}\right)^{n}\right]^{-s}+G_{1},
$$

where $T_{\mathrm{K}}$ is the Kondo temperature, $G_{0}$ is the Kondo conductance at $T=0 \mathrm{~K}$, and $G_{1}$ corresponds to a constant background conductance. The parameters $s$ and $n$ depend on the symmetry of the Kondo state. For the spin-1/2 Kondo effect, $s=0.22$ and $n=2$ are commonly used [SU(2) parameters], while Keller et al. have introduced $s=0.2$ and $n=3$ for the spin-orbital Kondo effect with SU(4) symmetry [17].

Figure 2(d) shows the temperature dependence of the conductance at $V_{\mathrm{sd}}=0$ and $\Delta E_{\mathrm{orb}}=0$ in the $1 e$ regime. Assuming that both the spin and orbital degeneracies contribute to the Kondo resonance, we use the $\mathrm{SU}(4)$ fitting parameters, and extract $T_{\mathrm{K}}=610 \mathrm{mK}\left(G_{0}=0.25 e^{2} / h, G_{1}=0.15\right.$ $\left.e^{2} / h\right)$ using Eq. (1). However, the standard parameters for the $\mathrm{SU}(2)$ Kondo effect also provide a good fit and would give a Kondo temperature of $T_{\mathrm{K}}=950 \mathrm{mK}\left(G_{0}=0.33 e^{2} / h\right.$, $\left.G_{1}=0.08 e^{2} / h\right)$, thus making it impossible to conclude the symmetry of the Kondo effect by the temperature dependence alone.

\section{B. Isolating the spin-1/2 Kondo effect}

Next, we isolate spin Kondo transport in the right QD by electrostatically detuning the system to the $(0,1)$ regime, and study its temperature dependence [Fig. 2(e)]. For the chosen sidegate voltages, $T_{\mathrm{K}}=350 \mathrm{mK}$ can be extracted using Eq. (1) and standard $\mathrm{SU}(2)$ parameters $\left(G_{0}=0.06 e^{2} / h\right.$, $\left.G_{1}=0.06 e^{2} / h\right)$. The fact that $G_{0} \ll 2 e^{2} / h$ can be explained 


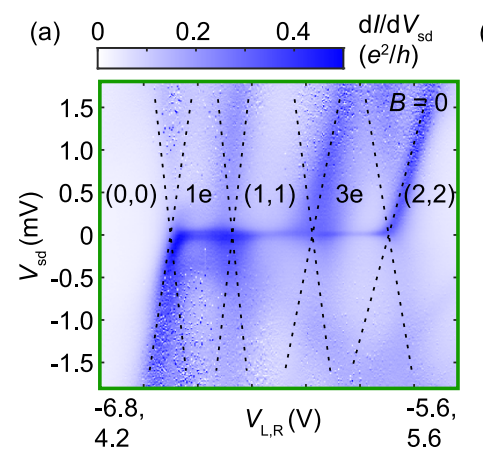

(b) 0.05

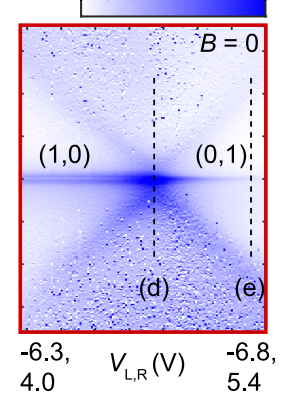

(g) 0.05
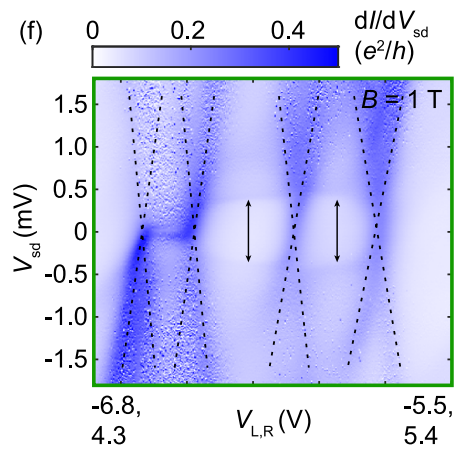

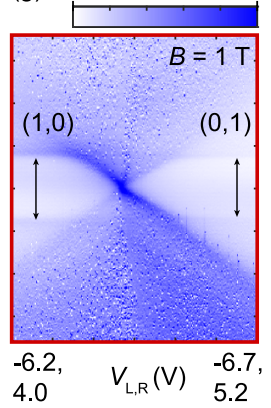

(c) $B=0, t=0$

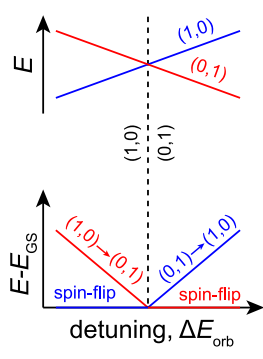

0.3 (h) $B=1 \mathrm{~T}, t=0$

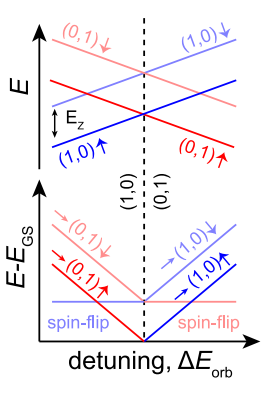

(d)

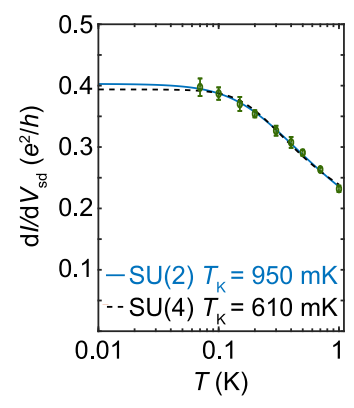

(i)

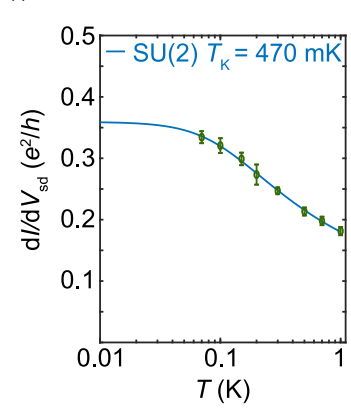

(e)

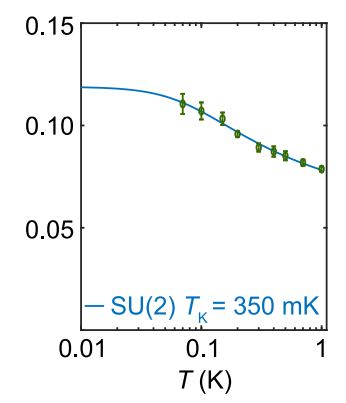

(j)

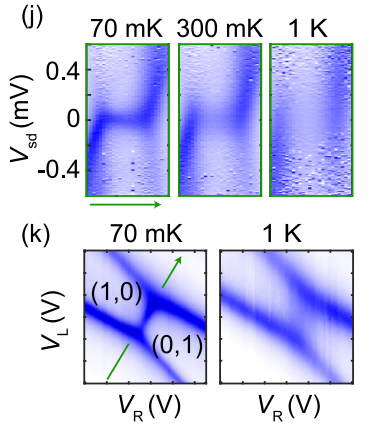

FIG. 2. Spin-orbital and isolated orbital Kondo transport in the one-electron regime. (a) Measurement of $d I / d V_{\text {sd }}$ vs $V_{\text {sd }}$ along the green gate vector indicated in Fig. 1(b) for $B=0$. A zero-bias peak can be observed in the $1 e, 2 e$, and $3 e$ regimes. (b) Corresponding measurement in the $1 e$ regime when detuning the orbitals along the red gate vector. The dashed lines represent the sidegate voltages where the temperature sweeps are performed. (c) Schematic representation of the electron energy levels and resulting onset of cotunneling when detuning the QD orbitals (assuming $t=0$ ). (d) Temperature dependence of the zero-bias conductance peak at orbital degeneracy. Blue and dashed black lines show the fit using Eq. (1) and the parameters for SU(2) and SU(4) Kondo scaling, respectively. (e) Temperature dependence and SU(2) Kondo fit of the zero-bias conductance peak in the $(0,1)$ state. (f)-(i) Corresponding measurements and schematic for $B=1 \mathrm{~T}$. The spin degeneracy is lifted, but the orbital degeneracy at zero detuning in the $1 e$ regime remains. (j) Same as (f), but for different temperatures (only $1 e$ regime). (k) Conductance as a function of sidegate voltages $\left(V_{\mathrm{sd}}=25 \mu \mathrm{V}\right)$ for $70 \mathrm{mK}$ and $1 \mathrm{~K}$ (1e regime).

by an asymmetry in the tunnel couplings to source and drain (Appendix D). In our QD system, the asymmetry can be due to a difference in barrier thickness and shape of the QDs.

\section{Isolating the orbital Kondo effect}

In order to unambiguously verify the orbital contribution to the Kondo effect at $\Delta E_{\text {orb }}=0$, we isolate the effect from spin Kondo transport using Zeeman spin splitting at $B=1 \mathrm{~T}$. The large effective $g$ factor $\left(g^{*}\right)$ of InAs ( -14.7 in bulk) facilitates lifting of the spin degeneracy by the Zeeman energy $E_{\mathrm{Z}}=g^{*} \mu_{\mathrm{B}} B$, where $\mu_{\mathrm{B}}$ is the Bohr magneton. In Fig. 2(f), a resulting gap is observed in the $2 e$ and $3 e$ regimes, where spin-flip transport by inelastic cotunneling is possible when the source-drain voltage is larger than the gap energy. Preliminarily, the absence of zero-bias peaks in the $2 e$ and $3 e$ regimes indicates that the observed Kondo peaks in Figs. 2(a) were due to a spin-related Kondo effect. However, in the $1 e$ region, the zero-bias peak remains, indicating that a different degeneracy is still present [38].

Detuning the orbitals along the red gate vector allows one to distinguish the Zeeman gap of the single QD orbitals from the onset of orbital cotunneling [Figs. 2(g) and 2(h)]. In the $(1,0)$ and $(0,1)$ regimes, we observe $E_{\mathrm{Z}} \approx 0.5 \mathrm{meV}$, corresponding to $g^{*} \approx 9$. The step in differential conductance, originating from the onset of orbital cotunneling transport, crosses in the Zeeman gap at $\Delta E_{\text {orb }}=0$. A zero-bias peak can be observed, which corresponds to the orbital Kondo effect. Temperature-dependent conductance data at this point [Fig. 2(i)] can be fitted with Eq. (1) and standard SU(2) parameters, resulting in $T_{\mathrm{K}}=470 \mathrm{mK}\left(G_{0}=0.28 e^{2} / h, G_{1}=0.08\right.$ $\left.e^{2} / h\right)$. Comparing the Kondo temperature for the spin-orbital Kondo resonance with that of the pure spin- $1 / 2$ or the pure orbital Kondo effect, we find a higher $T_{\mathrm{K}}$ and $G_{0}$ when both degeneracies are present, which is in agreement with a higher symmetry.

In Figs. 2(j) and 2(k), measurements at different temperatures are presented for $B=1 \mathrm{~T}$. The bias-dependent measurements of $d I / d V_{\mathrm{sd}}$ along the green gate vector in the $1 e$ regime [Fig. 2(j)] show that the zero-bias peak disappears with increasing temperature, while transport corresponding to sequential tunneling only exhibits a weak temperature dependence. Accordingly, the conductance line at orbital degeneracy strongly decays with temperature in Fig. 2(k).

We note that the presence of the orbital Kondo effect implies that the orbital quantum number is also a good quantum number in the leads (similar to spin). In this work, the results are discussed in the picture of coupled double QDs, where the orbital degeneracy is commonly attributed to zero tunnel coupling between the two QDs. An orbital Kondo resonance therefore requires two transport channels in the leads, each of them coupling predominantly only to one of the QDs. However, an orbital degeneracy would also be present if the two QDs are coupled into ringlike states 

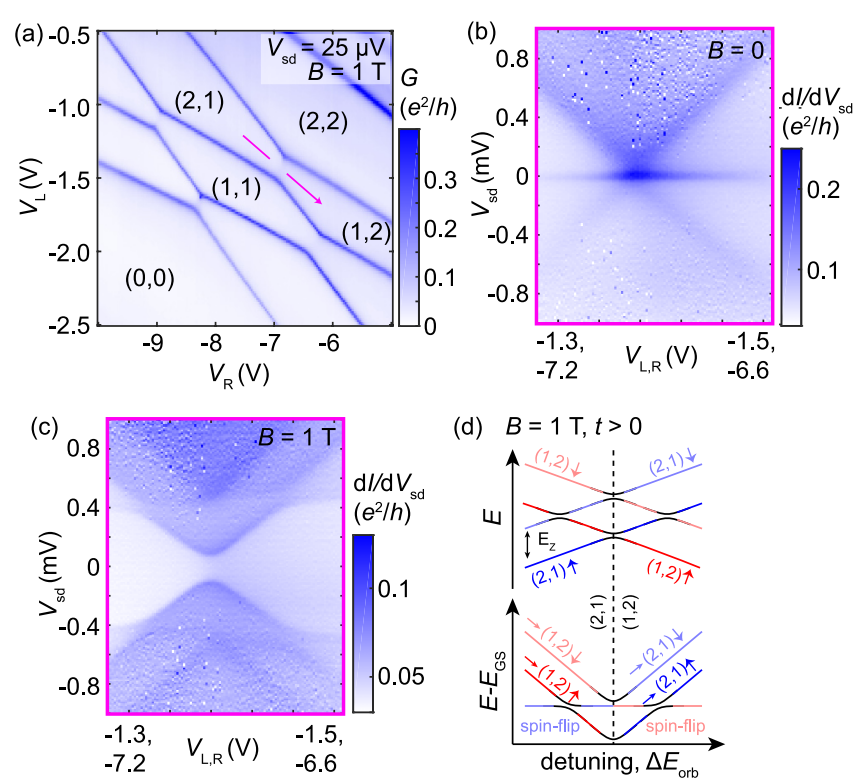

FIG. 3. The effect of a finite tunnel coupling between the QDs in another orbital crossing. (a) Overview measurement with the electron number on the left and right QD $\left(N_{\text {left }}, N_{\text {right }}\right)$ indicated. (b),(c) Measurement of $d I / d V_{\text {sd }}$ vs $V_{\text {sd }}$ recorded along the pink gate vector at $B=0 \mathrm{~T}$ and $B=1 \mathrm{~T}$. (d) Schematic representation of the energy levels and resulting onset of cotunneling when detuning the QD orbitals, assuming finite tunnel coupling between the two QD levels.

(cf. [35]), if the hybridization gap due to spin-orbit interaction and backscattering is smaller than the Kondo temperature [18,21,39].

\section{Effect of orbital hybridization}

In the following, we will discuss the effect of interdot tunnel coupling, based on data from the same device but from a different crossing of QD orbitals [Fig. 3(a)]. When detuning the orbitals along the pink gate vector for $B=0$, both a zerobias peak and a sloped step in differential conductance due to orbital cotunneling can be observed [Fig. 3(b)]. From this data, it appears as if the onset of orbital cotunneling crosses at zero bias for $\Delta E_{\text {orb }}=0$, similar to what has been shown in Fig. 2(b).

However, when the spin degeneracy is lifted $[B=1 \mathrm{~T}$, Fig. 3(c)], a finite gap at zero detuning is visible instead of an orbital Kondo resonance. This can be understood considering an avoided crossing due to interdot tunnel coupling, as schematically presented in Fig. 3(d). In this case, the hybridization gap is $\sim 70 \mu \mathrm{eV}$, corresponding to a temperature of $\sim 800 \mathrm{mK}$. Since the gap is comparable to the observed Kondo temperatures, it can only be detected by first lifting the spin degeneracy. A similar behavior, but with a much larger avoided crossing, is found for the $3 e$ regime of the crossing presented in Fig 1(b) (see Appendix C).

\section{E. Spin-1 Kondo transport}

Finally, we investigate Kondo transport in the $2 e$ regime by detuning the orbitals along the orange gate vector in Fig. 1(b). A zero-bias peak can be observed in the $(1,1)$ regime at
$B=0 \mathrm{~T}$ [Fig. 4(a)], indicating a Kondo resonance due to a degenerate ground state when each QD contains one electron. At $B=1 \mathrm{~T}$ [Fig. 4(b)], this degeneracy is lifted, resulting in a gap of $\sim 800 \mu \mathrm{eV}$ in the $(1,1)$ regime. A second-excited state can be observed that is $\sim 350 \mu \mathrm{eV}$ higher in energy than the first-excited state, which implies the existence of two-electron states due to hybridization, rather than noninteracting orbitals on each QD. To study the two-electron states in more detail, bias-dependent transport as a function of magnetic field was performed in the center of $(1,1)$, as shown in Fig. 4(c). The zero-bias peak Zeeman splits with $g^{*} \approx 7$, which is slightly different compared to $g^{*} \approx 9$ in the $1 e$ regime, likely due to the interaction with another orbital [40]. The second onset of cotunneling evolves parallel to the first-excited state. These observations are in agreement with a spin-triplet ground state and a spin-singlet excited state, with a singlet-triplet splitting of $J \approx 350 \mu \mathrm{eV}$, as schematically depicted in Fig. 4(d). The spin-triplet ground state could be explained by the formation of a quantum ring [41], which can form when two quantum dots couple in two points, as previously shown for this material system [35]. We find that the intensity of the zero-bias peak monotonically decreases both with $B$ field [Fig. 4(c)] and temperature [Fig. 4(d)], and can therefore be interpreted as an underscreened spin-1 Kondo effect $[15,42,43]$. The temperature scaling can be described by Eq. (1) and corresponds to $T_{\mathrm{K}}=780 \mathrm{mK}$
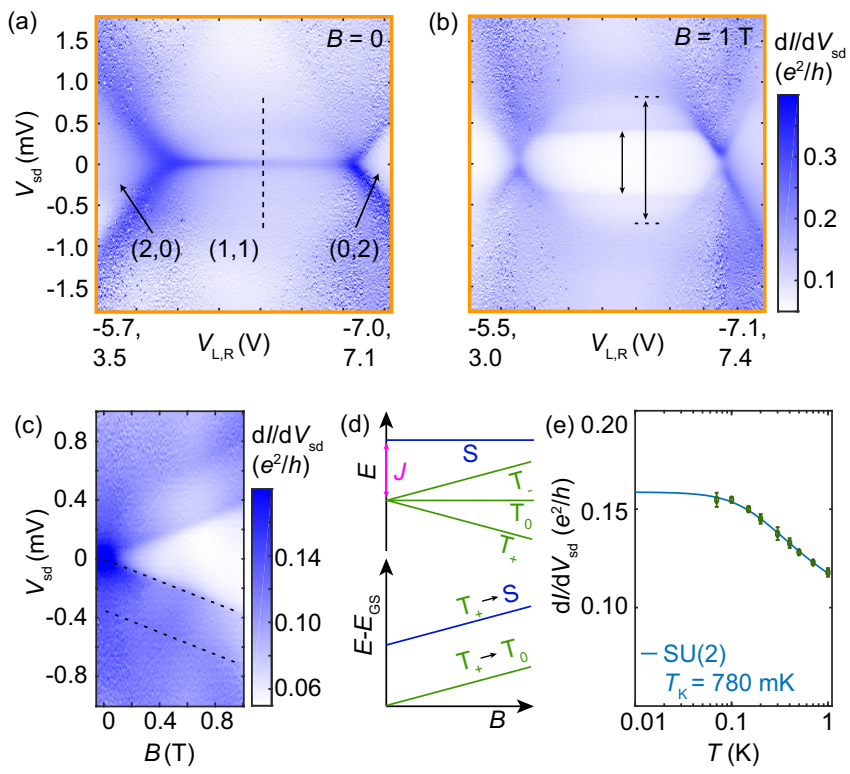

FIG. 4. Kondo transport in the two-electron regime. (a) Measurement of $d I / d V_{\mathrm{sd}}$ vs $V_{\mathrm{sd}}$ recorded along the orange gate vector at $B=0 \mathrm{~T}$, showing a Kondo resonance in $(1,1)$. (b) Corresponding measurement at $B=1 \mathrm{~T}$. The ground-state degeneracy is lifted and an additional onset of cotunneling is observed with an energy of $\sim 350 \mu \mathrm{eV}$ higher than the first-excited state. (c) Magnetic field sweep in the center of $(1,1)$. (d) Schematic representation of the $2 e$ state energies and resulting onset of cotunneling as a function of the $B$ field. The triplet GS Zeeman splits into $T_{+}, T_{0}$, and $T_{-}$. At finite $B$ field, $T_{+}$is the GS, and we observe transitions to $T_{0}$ and to the singlet state $S$. (e) Temperature dependence of the zero-bias conductance peak in the center of $(1,1)$ at $B=0 \mathrm{~T}$. 
$\left(G_{0}=0.07 e^{2} / h, G_{1}=0.08 e^{2} / h\right)$ if using standard $\mathrm{SU}(2)$ parameters.

\section{SUMMARY AND CONCLUSION}

In summary, we have studied Kondo transport in parallelcoupled QDs in InAs nanowires. In the $1 e$ regime, we observe the spin- $1 / 2$ Kondo effect, the combined spin-orbital Kondo effect, and the orbital Kondo effect when the spin degeneracy is lifted. We demonstrate that finite interdot tunnel coupling inhibits the orbital Kondo effect by hybridizing the orbitals, and emphasize that the presence of a small energy gap can only be detected when the spin degeneracy is selectively lifted. The $2 e$ regime exhibits a triplet ground state likely due to the formation of ringlike states, leading to a Kondo resonance at $B=0$. The possibility to isolate the different degeneracies makes this an ideal material system for studies of higher-symmetry Kondo effects.

\section{ACKNOWLEDGMENTS}

The authors thank I-J. Chen and J. Paaske for fruitful discussions. This work was carried out with financial support from the Swedish Research Council (VR), NanoLund, the Knut and Alice Wallenberg Foundation (KAW), and the Crafoord Foundation. H.P. thankfully acknowledges funding from the Swiss National Science Foundation (SNSF) via Early PostDoc Mobility Grant No. P2ELP2_178221.

\section{APPENDIX A: OVERVIEW CONDUCTANCE MEASUREMENT}

Figure 5(a) shows conductance $(G)$ for a wide range of sidegate voltages $\left(V_{\mathrm{L}}, V_{\mathrm{R}}\right)$. The overview measurement shows that honeycombs with no discernible hybridization can be observed for specific orbital crossings. We explain this by the formation of ringlike states when the orbitals are coupled in two points, as investigated in detail in Ref. [35]. The orbital crossing showing the spin-orbital Kondo effect (cf. Fig. 2) is highlighted with a red square, while the additional crossing where the orbital Kondo effect is suppressed due to a small hybridization gap (cf. Fig. 3) is highlighted with a green square. We note that the data presented in Fig. 3 were obtained at a backgate voltage of $V_{\mathrm{BG}}=-0.8 \mathrm{~V}$, while the overview here was obtained at $V_{\mathrm{BG}}=-1 \mathrm{~V}$. In this Appendix, we present additional measurements for the orbital crossing highlighted in red. The gate vectors which will be discussed in the following are indicated in the conductance measurement in Fig. 5(b) [same as Fig. 1(b)].

\section{APPENDIX B: EXTRACTION OF $T_{K}$ FROM THE VOLTAGE DEPENDENCE}

In the main paper, the red gate vector where the orbitals are detuned from the $(1,0)$ to the $(0,1)$ regime is discussed in great detail, and the Kondo temperature $\left(T_{\mathrm{K}}\right)$ is extracted from the temperature dependence of the zero-bias conductance peak. Alternatively, $T_{\mathrm{K}}$ can be determined from the source-drain voltage dependence of the zero-bias peak. Figure 1(c) shows differential conductance $\left(d I / d V_{\text {sd }}\right)$ versus the source-drain
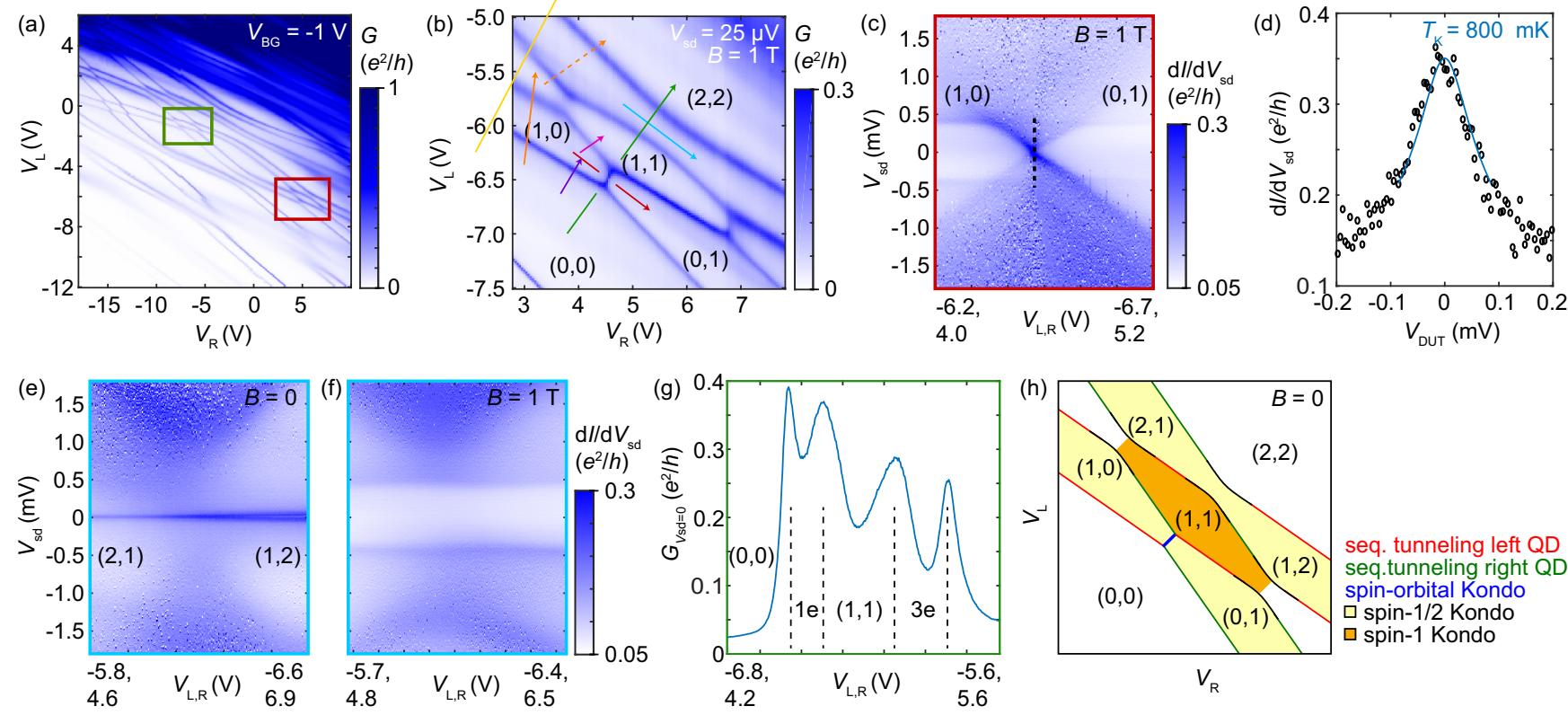

seq. tunneling left $Q D$ seq.tunneling right $Q D$ spin-orbital Kondo spin-1/2 Kondo $\square$ spin-1 Kondo

FIG. 5. (a) Conductance as a function of sidegate voltages. The red and green squares indicate the orbital crossings that are studied. (b) Magnified conductance plot of the red orbital crossing, where relevant gate vectors are indicated. (c) Differential conductance vs sourcedrain voltage recorded along the red gate vector at $B=1 \mathrm{~T}$. (d) Differential conductance as a function of voltage across the device measured at the sidegate voltages corresponding to the dashed line in (c). The data are fitted with Eq. (B1) [44]. (e),(f) Transport recorded in the $3 e$ regime along the cyan gate vector at $B=0 \mathrm{~T}$ and $B=1 \mathrm{~T}$, respectively. (g) Conductance $G$ at zero bias along the green gate vector. (h) Schematic representation of the orbital crossing and the different transport mechanisms at $B=0 \mathrm{~T}$. 
voltage $\left(V_{\text {sd }}\right)$ along the red gate vector at a magnetic field of $B=1 \mathrm{~T}$ [same as Fig. 2(g)]. Differential conductance as a function of applied voltage at the orbital degeneracy point (highlighted with a dashed line) is presented in Fig. 5(d). Note that the $x$ axis corresponds to the voltage applied across the device $\left(V_{\text {DUT }}\right)$, after subtracting the voltage drop across the series resistance of the amplifier and cryostat wiring and filters $\left(R_{\mathrm{S}}=16.5 \mathrm{k} \Omega\right)$. For the spin- $1 / 2$ Kondo effect, Pletyukhov et al. have introduced that the voltage dependence of the differential conductance can be described by

$$
d I / d V_{\mathrm{sd}}\left(V_{\mathrm{sd}}\right)=d I / d V_{\mathrm{sd}}(0)\left[1+\frac{v^{2}\left(2^{1 / s 1}-1\right)}{\left(1-b+b v^{s 2}\right)}\right]^{-s 1},
$$

with $s_{1}=0.32, \quad b=0.05, \quad s_{2}=1.26$ and $v=e\left(V_{\mathrm{sd}}-\right.$ $\left.V_{0}\right) /\left(k_{\mathrm{B}} T_{\mathrm{K}}^{*}\right)$. The differential conductance at zero bias $\left[d I / d V_{\mathrm{sd}}(0)\right]$, the Kondo temperature $\left(T_{\mathrm{K}}^{*}\right)$, and $V_{0}$ are fitting parameters [44]. Using this equation to fit our experimental data for the orbital Kondo resonance, and after substituting $T_{\mathrm{K}}^{*}=1.8 T_{\mathrm{K}}$, as suggested by Pletyukhov et al. [44], we extract a Kondo temperature of $T_{\mathrm{K}}=800 \mathrm{mK}$. We note that the Kondo temperature extracted from the voltage dependence is slightly higher compared to the one obtained by the temperature-dependent data.

\section{APPENDIX C: TRANSPORT IN THE $3 e$ REGIME}

In the main paper, we showed that the orbital Kondo effect is absent if tunnel coupling between the two QDs leads to an avoided level crossing at $\Delta E_{\text {orb }}=0$ (cf. Fig. 3). In order to illustrate that lifting the spin degeneracy can be key to detecting a small hybridization gap, we presented data from a different orbital crossing [highlighted with a green square in Fig. 5(a)]. Here, we show transport measurements for the $3 e$ regime of the orbital crossing highlighted with a red square in Fig. 5(a). Figures 5(e) and 5(f) show $d I / d V_{\text {sd }}$ as a function of $V_{\text {sd }}$ for $B=0$ and $B=1 \mathrm{~T}$, when detuning the orbitals along the cyan gate vector. We observe a relatively large hybridization gap, which can be seen both with and without magnetic field. This finding shows that adding electrons to the QD orbitals slightly alters the tunnel coupling between them. In the $1 e$ regime, the gate voltages were tuned in order to have the orbital Kondo effect (which requires the absence of a hybridization gap). In the $2 e$ regime, the two electrons strongly interact, resulting in two-electron states (cf. Fig 4). Finally, in the $3 e$ regime, the electrons also strongly interact, which we interpret as a gradual increase in tunnel coupling strength with particle number. Figure $5(\mathrm{~g})$ shows the conductance at zero bias along the green gate vector [line cut at $V_{\text {sd }}=0$ in Fig. 2(a)]. The sequential tunneling peaks are clearly visible, indicating that the sample is not in the mixed valence regime for sidegate voltages in the center of the valleys [23]. This will be confirmed by estimating the tunnel coupling in Appendix D.

Based on our observations, the different mechanisms leading to transport through this particular orbital crossing are summarized in Fig. 5(h): The red and green lines correspond to sequential tunneling through the left and right QD, respectively. The spin-1/2 Kondo effect is observed in the $(0,1)$, $(1,0),(1,2)$, and $(2,1)$ regimes (yellow shading). In the $1 e$ regime, the tunnel coupling between the two QDs is zero,
TABLE I. Approximate values of the charging energy and the QD lever arms.

\begin{tabular}{lccccc}
\hline \hline & $\begin{array}{c}E_{c} \\
(\mathrm{meV})\end{array}$ & $\begin{array}{c}\alpha_{V \mathrm{~L} / \mathrm{L}} \\
(\mathrm{meV} / \mathrm{V})\end{array}$ & $\begin{array}{c}\alpha_{V \mathrm{R} / \mathrm{L}} \\
(\mathrm{meV} / \mathrm{V})\end{array}$ & $\begin{array}{c}\alpha_{V \mathrm{~L} / \mathrm{R}} \\
(\mathrm{meV} / \mathrm{V})\end{array}$ & $\begin{array}{c}\alpha_{V \mathrm{R} / \mathrm{R}} \\
(\mathrm{meV} / \mathrm{V})\end{array}$ \\
\hline $\begin{array}{l}\text { Left QD } \\
\text { Right QD }\end{array}$ & 6 & 14 & 4 & & \\
\hline \hline
\end{tabular}

leading to the spin-orbital Kondo effect at orbital degeneracy (blue line). In the $(1,1)$ regime, a Kondo resonance is found due to a degenerate triplet ground state (orange shading). No orbital Kondo effect is found between the $(2,0)-(1,1)-(0,2)$ regimes, explained by a small spin-orbit-induced mixing [see small avoided crossings in Fig. 4(b)].

\section{APPENDIX D: EXTRACTION OF THE LEVER ARMS AND TUNNEL COUPLING}

In Figs. 6(a) and 6(b), we present $d I / d V_{\text {sd }}$ versus $V_{\text {sd }}$ for gate vectors through the unperturbed levels of the left and right QD [corresponding to the solid and dashed orange vectors in Fig. 5(b)] at $B=0 \mathrm{~T}$. A zero-bias peak can be observed due to the spin-1/2 Kondo effect in the $(1,0)$ and $(2,1)$ regimes, when the electron population on one of the QDs is odd. At $B=1 \mathrm{~T}$ [Fig. 6(c)], the spin degeneracy is lifted, resulting in a Zeeman gap. From the height of the Coulomb diamonds in Figs. 6(a)-6(c), the charging energy $E_{\mathrm{c}}$ of the QDs can be extracted. The width of the Coulomb diamonds is then used to calculate the lever arms of the QDs with respect to each of the two sidegate voltages. We note that the Coulomb diamonds were recorded by sweeping both sidegates simultaneously [as indicated on the $x$ axis of Figs. 6(a)-6(c)], and we used the slope of conductance lines in Fig. 5(b) in order to calculate an effective gate vector which depends only on one sidegate voltage. The relevant parameters for both QDs are summarized in Table I. Here, $\alpha_{V \mathrm{~L} / \mathrm{L}}$ denotes the lever arm of the left QD (L) with respect to the left sidegate voltage $V_{\mathrm{L}}$, and the other lever arms are labeled correspondingly.

Next, we extract the tunneling coupling $(\Gamma)$ to the two QDs. This is relevant since, strictly speaking, the empirical equation to calculate $T_{\mathrm{K}}$ from the temperature dependence is only valid as long as $\epsilon_{0} / \Gamma<-0.5$, where $\epsilon_{0}$ is the energy difference of the nearest lower-energy state relative to the Fermi level of the contacts [1].

$\Gamma$ can be estimated from the conductance due to sequential tunneling through a QD level. At low source-drain voltage and low temperature $\left(k_{\mathrm{B}} T \ll \Gamma\right)$, the conductance $G$ as a function of gate voltage $\left(V_{\text {gate }}\right)$ can be described by a Lorentzian,

$$
G=\frac{G_{\max }}{1+\left(2 \Delta V_{\text {gate }} / \Gamma\right)^{2}}+c
$$

where $G_{\max }$ is the peak conductance, $\Delta V_{\text {gate }}=V_{\text {gate }}-V_{\text {gate }, 0}$ is the detuning from the QD resonance centered at $V_{\text {gate }, 0}, \Gamma$ is the full width at half maximum (FWHM) of the Lorentzian, and $c$ is a constant offset. The FWHM corresponds to the sum of the tunnel couplings to source and drain, $\Gamma=\Gamma_{\mathrm{S}}+\Gamma_{\mathrm{D}}$. Figures 6(d) and 6(e) show line cuts through an orbital of the left and right QD, corresponding to the purple and pink gate vector in Fig. 5(b). We note that the gate vectors were 

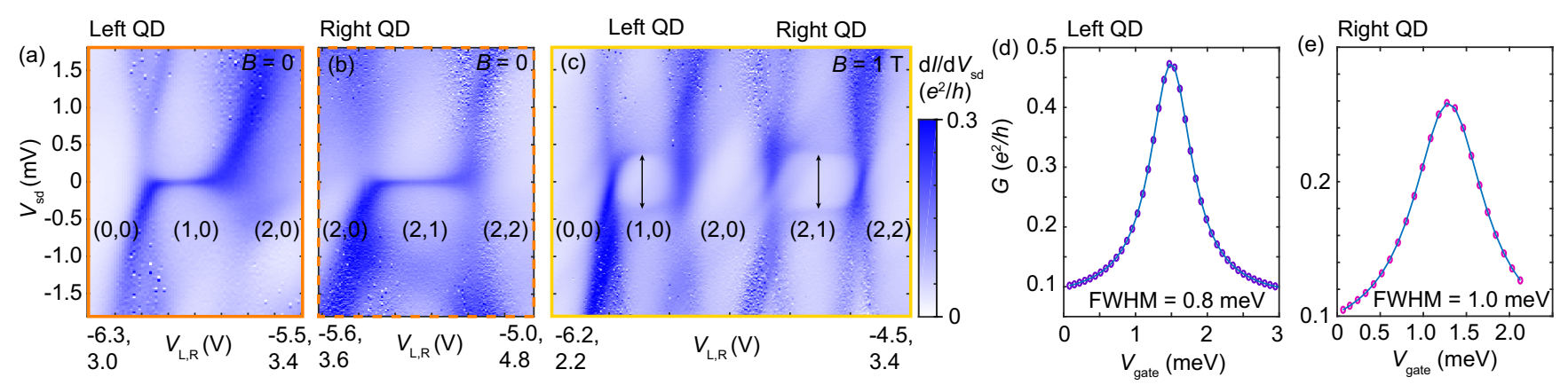

FIG. 6. (a) Differential conductance vs source-drain voltage as a function of gate vector through the unperturbed left and right QD levels [solid and dashed orange gate vector in Fig. 5(b)] for $B=0 \mathrm{~T}$. (c) Corresponding measurement along the yellow gate vector for $B=1 \mathrm{~T}$. (d), (e) Conductance as a function of gate voltage for sequential tunneling through the left and right QD [purple and pink gate vector in Fig. 5(b)]. The data is fitted using Eq. (D1) to extract $\Gamma$.

chosen such that they are perpendicular to the conductance lines of the QD levels, after having converted the sidegate voltages into energies using the lever arms. Using Eq. (D1), $\Gamma \approx 0.8 \mathrm{meV}$ and $\Gamma \approx 1 \mathrm{meV}$ can be extracted for the left and right $\mathrm{QD}$, respectively. We note that the sequential tunneling lines were measured at $V_{\mathrm{sd}}=25 \mu \mathrm{eV}$, leading to an additional broadening of the peak. The calculated $\Gamma$ therefore can be considered an upper bound. For the orbital Kondo effect, the measurement was done at orbital degeneracy in the $1 e$ in the middle of the two charge state resonances [see Fig. 2(a)]. We therefore have $\epsilon_{0}=-0.5 U_{1,2}=-1.5 \mathrm{meV}$, where $U_{1,2}=3 \mathrm{meV}$ is the interdot Coulomb energy. With $\epsilon_{0} / \Gamma=-1.5<-0.5$, transport is in the Kondo regime and the empirical equation to extract the Kondo temperature from the temperature dependence is therefore valid.

Finally, we extract the asymmetry $\Gamma_{\mathrm{S}} / \Gamma_{\mathrm{D}}$ for the two QDs based on the sequential tunneling conductance in Figs. 6(d) and 6(e). Using $G_{\max }=\left(4 \Gamma_{\mathrm{S}} \Gamma_{\mathrm{D}}\right) /\left(\Gamma_{\mathrm{S}}+\Gamma_{\mathrm{D}}\right)^{2} e^{2} / h$, we extract $\Gamma_{\mathrm{S}} / \Gamma_{\mathrm{D}} \approx 20$ and $\Gamma_{\mathrm{S}} / \Gamma_{\mathrm{D}} \approx 8$ for the left and right $\mathrm{QD}$, respectively. We note that an estimation of the asymmetry based on $G_{0}$ in the Kondo regime would be less accurate since the electron temperature is comparable to $T_{\mathrm{K}}$ [45]. The asymmetry of the tunnel couplings of each QD also depends on the sidegate voltages due to a change of confinement potential.
[1] D. Goldhaber-Gordon, J. Göres, M. A. Kastner, H. Shtrikman, D. Mahalu, and U. Meirav, Phys. Rev. Lett. 81, 5225 (1998).

[2] S. M. Cronenwett, T. H. Oosterkamp, and L. P. Kouwenhoven, Science 281, 540 (1998).

[3] J. Schmid, J. Weis, K. Eberl, and K. v. Klitzing, Phys. B: Condens. Matter 256-258, 182 (1998).

[4] J. Schmid, J. Weis, K. Eberl, and K. v. Klitzing, Phys. Rev. Lett. 84, 5824 (2000).

[5] A. Kogan, G. Granger, M. A. Kastner, D. Goldhaber-Gordon, and H. Shtrikman, Phys. Rev. B 67, 113309 (2003).

[6] J. Paaske, A. Rosch, P. Woelfle, N. Mason, C. M. Marcus, and J. Nygard, Nat. Phys. 2, 460 (2006).

[7] S. Tarucha, D. G. Austing, Y. Tokura, W. G. van der Wiel, and L. P. Kouwenhoven, Phys. Rev. Lett. 84, 2485 (2000).

[8] M. Pustilnik, Y. Avishai, and K. Kikoin, Phys. Rev. Lett. 84, 1756 (2000).

[9] S. Sasaki, S. De Franceschi, J. Elzerman, W. van der Wiel, M. Eto, S. Tarucha, and L. Kouwenhoven, Nature (London) 405, 764 (2000).

[10] D. Giuliano, B. Jouault, and A. Tagliacozzo, Phys. Rev. B 63, 125318 (2001).

[11] W. G. van der Wiel, S. De Franceschi, J. M. Elzerman, S. Tarucha, L. P. Kouwenhoven, J. Motohisa, F. Nakajima, and T. Fukui, Phys. Rev. Lett. 88, 126803 (2002).

[12] S. J. Chorley, M. R. Galpin, F. W. Jayatilaka, C. G. Smith, D. E. Logan, and M. R. Buitelaar, Phys. Rev. Lett. 109, 156804 (2012).
[13] W. Liang, M. Bockrath, and H. Park, Phys. Rev. Lett. 88, 126801 (2002).

[14] C. H. L. Quay, J. Cumings, S. J. Gamble, R. dePicciotto, H. Kataura, and D. Goldhaber-Gordon, Phys. Rev. B 76, 245311 (2007).

[15] N. Roch, S. Florens, T. A. Costi, W. Wernsdorfer, and F. Balestro, Phys. Rev. Lett. 103, 197202 (2009).

[16] D. Krychowski and S. Lipiński, Phys. Rev. B 93, 075416 (2016).

[17] A. J. Keller, S. Amasha, I. Weymann, C. P. Moca, I. G. Rau, J. A. Katine, H. Shtrikman, G. Zaránd, and D. GoldhaberGordon, Nat. Phys. 10, 145 (2013).

[18] T. Delattre, L. G. Herrmann, P. Morfin, J. Berroir, G. Fève, B. Plaçais, D. C. Glattli, M. Choi, C. Mora, and T. Kontos, Nat. Phys. 5, 208 (2009).

[19] M. Ferrier, T. Arakawa, T. Hata, R. Fujiwara, R. Delagrange, R. Deblock, R. Sakano, A. Oguri, and K. Kobayashi, Nat. Phys. 12, 230 (2015).

[20] M. Ferrier, T. Arakawa, T. Hata, R. Fujiwara, R. Delagrange, R. Deblock, Y. Teratani, R. Sakano, A. Oguri, and K. Kobayashi, Phys. Rev. Lett. 118, 196803 (2017).

[21] P. Jarillo-Herrero, J. Kong, H. S. J. v. d. Zant, and C. Dekker, Nature (London) 434, 484 (2005).

[22] A. Makarovski, A. Zhukov, J. Liu, and G. Finkelstein, Phys. Rev. B 75, 241407(R) (2007).

[23] A. Makarovski, J. Liu, and G. Finkelstein, Phys. Rev. Lett. 99, 066801 (2007). 
[24] G. P. Lansbergen, G. C. Tettamanzi, J. Verduijn, N. Collaert, S. Biesemans, M. Blaauboer, and S. Rogge, Nano Lett. 10, 455 (2010).

[25] G. C. Tettamanzi, J. Verduijn, G. P. Lansbergen, M. Blaauboer, M. J. Calderón, R. Aguado, and S. Rogge, Phys. Rev. Lett. 108, 046803 (2012).

[26] U. Wilhelm, J. Schmid, J. Weis, and K. Klitzing, Phys. E (Amsterdam, Neth.) 14, 385 (2002).

[27] A. W. Holleitner, A. Chudnovskiy, D. Pfannkuche, K. Eberl, and R. H. Blick, Phys. Rev. B 70, 075204 (2004).

[28] S. Sasaki, S. Amaha, N. Asakawa, M. Eto, and S. Tarucha, Phys. Rev. Lett. 93, 017205 (2004).

[29] S. Amasha, A. J. Keller, I. G. Rau, A. Carmi, J. A. Katine, H. Shtrikman, Y. Oreg, and D. Goldhaber-Gordon, Phys. Rev. Lett. 110, 046604 (2013).

[30] Y. Okazaki, S. Sasaki, and K. Muraki, Phys. Rev. B 84, 161305(R) (2011).

[31] M. Nilsson, L. Namazi, S. Lehmann, M. Leijnse, K. A. Dick, and C. Thelander, Phys. Rev. B 93, 195422 (2016).

[32] M. Nilsson, I.-J. Chen, S. Lehmann, V. Maulerova, K. A. Dick, and C. Thelander, Nano Lett. 17, 7847 (2017).

[33] M. Nilsson, F. V. Boström, S. Lehmann, K. A. Dick, M. Leijnse, and C. Thelander, Phys. Rev. Lett. 121, 156802 (2018).

[34] C. Thelander, M. Nilsson, F. Viñas Boström, A. Burke, S. Lehmann, K. A. Dick, and M. Leijnse, Phys. Rev. B 98, 245305 (2018).

[35] H. Potts, I.-J. Chen, A. Tsintzis, M. Nilsson, S. Lehmann, K. A. Dick, M. Leijnse, and C. Thelander, Nat. Commun. 10, 5740 (2019).
[36] We also note a finite splitting of the spin-1/2 Kondo resonance in the $(1,0)$ regime. This could imply spin polarization of the electrons in the InAs nanowire segments that act as source and drain due to the Ni contacts, similar to observations in carbon nanotubes with $\mathrm{Ni}$ contacts [46].

[37] T. A. Costi, A. C. Hewson, and V. Zlatic, J. Phys.: Condens. Matter 6, 2519 (1994).

[38] We note that the small gap in the zero-bias peak in the $1 e$ regime can be explained by the gate vector being not exactly aligned with the degeneracy of the QD levels.

[39] J. Paaske, A. Andersen, and K. Flensberg, Phys. Rev. B 82, 081309(R) (2010).

[40] S. Csonka, L. Hofstetter, F. Freitag, S. Oberholzer, C. Schönenberger, T. S. Jespersen, M. Aagesen, and J. Nygård, Nano Lett. 8, 3932 (2008).

[41] B. Baxevanis and D. Pfannkuche, Coulomb interaction in finitewidth quantum rings, in Physics of Quantum Rings (Springer, Berlin, 2014), Chap. 15, pp. 381-408.

[42] M. Pustilnik and L. I. Glazman, Phys. Rev. Lett. 87, 216601 (2001).

[43] A. Posazhennikova and P. Coleman, Phys. Rev. Lett. 94, 036802 (2005).

[44] M. Pletyukhov and H. Schoeller, Phys. Rev. Lett. 108, 260601 (2012).

[45] M. Pustilnik and L. Glazman, J. Phys.: Condens. Matter 16, R513 (2004).

[46] J. R. Hauptmann, J. Paaske, and P. E. Lindelof, Nat. Phys. 4, 373 (2008). 